\title{
Part XII
}

\section{Addresses of participants}

Houshang Ardavan, Institute of Astronomy, University of Cambridge, Madingley Road, Cambridge, CB3 OHA UNITED KINGDOM Tel: 44-223-351721 Fax: 44-223-352941 Telex: 817297ASTRONG

Jonathan Arons, Department of Astronomy, 601 Campbell Hall, University of California at Berkeley, Berkeley, CA, 94920 USA Tel: 415-642-4730 Fax: 415-641-3411

Email: arons@astroplasma.berkeley.edu

Estelle Asseo, Centre de Physique Theorique de l'Ecole Polytechnique, Plateau de Palaiseau, 91128, Cedex, FraNCE Fax: 33-1-69-41-81-71 Telex: ECOLEX 601596F

Matthew Bailes, Nuffield Radio Astronomy Laboratories, Jodrell Bank, Macclesfield, Cheshire, SK11 9DL UNITED KINGDOM Tel: 44-477-71321 Fax: 44-477-71618 Telex: 36149 Email: JDBQuk.ac.man.jb.star

Vasily S. Beskin, P. N. Lebedev Physical Institute, Academy of Sciences, 117924, Moscow, RussiA Tel: 1244616 Fax: 1358533 Telex: 411479 NEOD SU

Dipankar Bhattacharya, Raman Research Institute, Bangalore, 560080 INDIA Tel: 91812340122 Fax: 91812340492 Telex: 8452671 RRI IN Email: rri@vigyan.ernet.in

James D. Biggs, Nuffield Radio Astronomy Laboratories, Jodrell Bank, Macclesfield, Cheshire, SK11 9DL UNITED KINGDOM Tel: 047771321 Fax: 047771618 Telex: 36149 Email: jdb@uk.ac.man.jb.star

Gennadii S. Bisnovatyi-Kogan, Institute for Space Research, Academy of Sciences, Profsojuznaya 88, 117810, Moscow, Russin Tel: 230-255-85 Fax: 7-095-310 Telex: 411498 STAR SU

Claes-Ingvar Björnsson, Stockholm Observatory, S-133 36, Saltsjöbaden, SwEDEN Tel: 08-7170380 Fax: 08-7174719 Telex: SOBSERV S 12972 Email: bjornsso@astro.su.se

S. V. Bogovalov, Moscow Engineering-Physical Institute, Kashirskoe Shosse 31, 115409, Moscow, RussiA

Valentín Boriakoff, Worcester Polytechnic Institute, Electrical Engineering Department, 306 Atwater Kent Laboratory, Worcester, MA, 01609 USA Tel: 508-831-5061 Fax: 508-831-5483 Email: boriakof@ee.evpi.edu

Yuri M. Bruk, Radioastronomical Institute, Krasnoznamyonnaya St. 4, 310002, Kharkov UKRAINE Tel: 47-85-58

Abraham C. L. Chian, Institute for Space Research (INPE/CNPq), Sao Jose dos Campos, Sao Paulo, BrazIL Tel: $123-229977$ ext. 426 Fax: 123-21-8743 Telex: 123-3530INPE BR Email: inpedge $@$ brfapesp.bitnet

James M. Cordes, Astronomy Department, Cornell University, Space Science Building, Ithaca, NY, 14853 USA Tel: 607-255-0608 Fax: 607-255-8803 Telex: 932454 Email: cordes@astrosun.tn.cornell.edu
Nichi D'Amico, Istituto Radioastronomia CNR, Via Della Fiorentina, 40060 Villa Vontana (BO), ITALY Tel: 39-51-696.5041 Fax: 39-51-696.51.05

Marek Demianski, Instytut Fizyki Teoretycznej, Uniwersytet Warszawski, ul. Hoza 69, Warszawa, Poland Tel: 48-22-21-27-00 Fax: 48-22-21-94-75

Wojciech Dziembowski, CAMK, Bartycka 18, Warszawa, 00-716 PolAND

Roger S. Foster, Naval Research Laboratory, Code 4200, Washington, DC, 20375-5000 USA Tel: 202-767-0669 Email: foster@rira.nrl.navy.mil

Jeffrey Foster Friedman, Universidad de Puerto Rico, Departmento de Fisica R. U. M, Mayaguez, PR, 00708 USA Tel: 809-834-2020x3542 Email: jeff@naic.bitnet

Janusz A. Gil, Astronomical Centre and Institute of Physics, Pedagogical University, Lubuska 2, 65-265, Zielona Góra, Poland Tel: 011-48 6866385 fax: 486866385 telex: 0433467 wsp pl

Stan Gorgolewski, Katedra Radioastronomii, Piwnice k/Torunia, 87-148, Lysomice, PolAND

Dennison Martin Gould, Nuffield Radio Astronomy Laboratories, Jodrell Bank, Macclesfield, Cheshire, SK11 9DL UNITED KINGDOM Tel: 44-477 71321 Fax: 44-477-71618 Telex: 36149 Email: dmgg@uk.ac.man.js.star

Alexander V. Gurevich, P. N. Lebedev Physical Institute, Academy of Sciences, 117924, Moscow, RussiA Tel: 1326171 Telex: 411479 NEODSU

Ismail O. Guseinov, Institute of Physics, Narimanov Avenue 33, 370143, Baku AzerbaiJan

Peter (Pip) A. Hamilton, Department of Physics, University of Tasmania, Hobart, Australia Tel: 6102-20-2419 Fax: 6102-20-2410 Telex: AA58150

Pawel Haensel, CAMK, Bartycka 18, Warszawa, 00-716 Poland Tel: 411086 Telex: 813978 Email: wojtek@aavobs.dk

Han Ji Ling, Department of Physics, Beijing Normal University, Beijing, PRC

Timothy H. Hankins, Department of Physics and Astronomy, NMIMT, Socorro, NM, 87801 USA Tel: 505-835-7326 Fax: 505-835-7027 Telex: 9109881710 Email: thankins@nrao.edu

Heinz Herold, Theoretical Astrophysics, University of Tübingen, Auf der Morgenstelle 10, D-7400, Tübingen, GermaNY Tel: 49-7071-292043 Fax: 49-7071-295400 Telex: 7262867 UTNA D Email: herold@tat.physik.uni-tuebingen.de

Antony Hewish, Mullard Radio Astronomy Observatory, Cavendish Laboratory, Madingley Road, Cambridge, CB3 OHE UNITED KINGDOM Tel: 0223-337296 Fax: 0223-63263 Telex: 81292

Yacov N. Istomin, P. N. Lebedev Physical Institute, Academy of Sciences, 117924, Moscow, RUSSIA Tel: 132-61-71 Telex: 411479 NEOD SU 
Vera A. Izvekova, Radio Astronomy Department, Lebedev Physical Institute, Academy of Sciences, 117924, Moscow, RussiA Tel: 9233558 Telex: 411479 NEOD SU

J. A. Javakhishvili, Tbilisi State University, I. Chavchavadze Avenue 3, 380028, Tbilisi, GeorgiA

Axel Jessner, Max Planck Institut für Radioastronomie, Auf dem Hügel 69, D-5300, Bonn, Germany Tel: 02257-301-18

Email: psi\%4505001007::JESSNER

Roman Juszkiewicz, CAMK, Bartycka 18, Warszawa, Poland

R. C. Kapoor, Indian Institute of Astrophysics, Bangalore, 560034 INDIA Tel: 530672 Telex: 8452763

Alexander Z. Kazbegi, Abastumani Astrophysical Observatory, Pavlov Street 2a, 380060, Tbilisi, Georgin Tel: 375226 Telex: 212234 APOGEI

Wlodek Kluzniak, Department of Physics, Columbia University, Pupin Physics Laboratories, New York, NY, 10027 USA Tel: 212-854 4451 Fax: 212-316 9504

Vitalii V. Kocharovsky, Institute of Applied Physics, Academy of Sciences, Gor'kii, RussiA Tel: 66-18-78 Telex: 412580 FIZIK SU

Vladimir V. Kocharovsky, Institute of Applied Physics, Academy of Sciences, Gor'kii, Russia Tel: 38-4.3-85 Telex: 412580 FIZIK SU

Yuri D. Kotcov, Moscow Engineering-Physical Institute, Kashirskoe Shosse 31, 115409, Moscow, RussiA

Yuri A. Kovalev, Institute for Space Research, Academy of Sciences, Profsojuznaya 88, 117810, Moscow, RussiA Tel: 333-21-89 Fax: 011-7-95-310-7023 Telex: 411498 A STAR SU

K. S. Kozak, Institute for Space Research, Academy of Sciences, Profsojuznaya 88, 117810 , Moscow, RussiA Tel: 333-21-89 Fax: 011-7-95-310-7023 Telex: 411498 A STAR SU

Wolfgang Kundt, Institut für Astrophysik, Auf dem Hügel 71, D-5300, Bonn, GERMANY

Tel: 0228-73-3653 Fax: 49-228-525-229

Telex: 0886440 MPIFR D

Arcadii D. Kuz'min, Radio Astronomy Department, Lebedev Physical Institute, Academy of Sciences, 117924, Moscow, Russia Telex: 411479 NEOD SU

Oleg A. Kus'min, Institute for Space Research, Academy of Sciences, Profsojuznaya 88, 117810, Moscow, Russia Tel: 333-21-89

Fax: 011-7-05-310-7023

Telex: 411498 A STAR SU

Li Ting Yi, Zhenjiang Shipbuilding Institute, P. O. Box 414, Zhenjiang, Jiangsu Prov., PRC

Diumber G. Lominadze, Abastumani Astrophysical Observatory, Georgian Academy of Sciences, Rustaveli Avenue 52, 380008, Tbilisi, GeorgiA Fax: 7-095-310-7023 c/o L. M. Zelenyi

Andrew G. Lyne, Nuffield Radio Astronomy Laboratories, Jodrell Bank, Macclesfield, Cheshire, SK11 9DL UNITED KINGDOM Tel: 0477-71321 Fax: 0477-71618 Telex: 36149 Email: AGL@US.AC.MAN.JB.STAR

Yuri E. Lyubarsky, Radioastronomical Institute, Krasnoznamyonnays St. 4, 310002, Kharkov UKRAINE
George Z. Machabeli, Abastumani Astrophysical Observatory, Pavlov Street 2a, 380060, Tbilisi, GeORGIA Tel: 375226 Telex: 212234 APOGEI

Valerii M. Malofeev, Radio Astronomy Department, P. N. Lebedev Physical Institute, Academy of Sciences, 117924, Moscow, RUSSIA Tel: 9233558 Telex: 411479 NEOD SU

Igor F. Malov, Radio Astronomy Department, P. N. Lebedev Physical Institute, Academy of Sciences, 117924, Moscow, Russia Tel: 9233558 Telex: 411479 NEOD SU

Richard N. Manchester, Radiophysics Division, CSIRO, P.O. Box 76, Epping, 2121, N.S.W. Australia Tel: 61-02-868 0222 Fax: 61028680310 Telex: 26230 ASTRO Email: rmanches@rpepping.oz.a

Peter M. McCulloch, Department of Physics, University of Tasmania, Hobart, 7001 AUSTRALIA Tel: 6102-20-2420 Fax: 6102-20-2410 Telex: AA58150

Mark M. McKinnon, Department of Physics, New Mexico Institute of Mining and Technology, Socorro, NM, 87801 USA Tel: 505-835-7274 Fax: 505-835-7027 Telex: 9109881710 Email: mmckinno@nrao.edu

George I. Melikidze, Abastumani Astrophysical Observatory, Pavlov Street 2a, 380060, Tbilisi, Georgin Tel: 375226 Telex: 212234 APOGEI

Donald B. Melrose, School of Physics, University of Sydney, Sydney, NSW, 2006 AUSTRalia Tel: 02-6922537 Fax: 02-6602903 Telex: AA26169 UNISYD Email: melrose@extro.ucc.su.oz

Leon Mestel, Astronomy Centre, University of Sussex, Falmer, Brighton, East Sussex, BN1 9QH UNITED KINGDOM Tel: 44-0273-678071 Fax: 44-0273-678097 Telex: 877159 BHVTXS G Email: mafb1\%uk.ac.sussex.closter

F. Curtis Michel, Space Physics and Astronomy Department, Rice University, Houston, Texas, 77001 USA Tel: 713-527-4925 Fax: 713-285-5143 Telex: 5564 Email: FCM@curt.rice.edu Email: 128.42.10.4

Alexander G. Muslimov, A. F. Ioffe Phys-Technica! Institute, Academy of Sciences, 194021, St. Petersburg, RussiA Tel: 247.93.26 Telex: 121.453 FTIAN SU

Ramesh Narayan, Steward Observatory, University of Arizona, Tucson, AR, 85721 USA Tel: 602-621-2560 Fax: 602-621-1532 Telex: 467175

Alexander Yu. Novikov, Institute for Space Research, Academy of Sciences, Profsojuznaya 88, 117810, Moscow, Russia Tel: 333-21-89

Fax: 011-7-95-310-7023

Telex: 411-498A STAR SU

Leszek Nowakowski, Department of Physics, University of Puerto Rico, Mayaguez Campus, Mayaguez, PR, 00708 USA

Yu. P. Ochelkov, Institute for Space Research, Academy of Sciences, Profsojusnaya 88, 117810, Moscow, RUSsiA

Haki Ögelman, Max Planck Institut für Extraterrestriche Physik, Giessenbachstrasse, D-8046, Garching bei München Germany Tel: 49 (89) 3299833 Fax: $49(89) 3299569$ Telex: 5215845 xterrd 


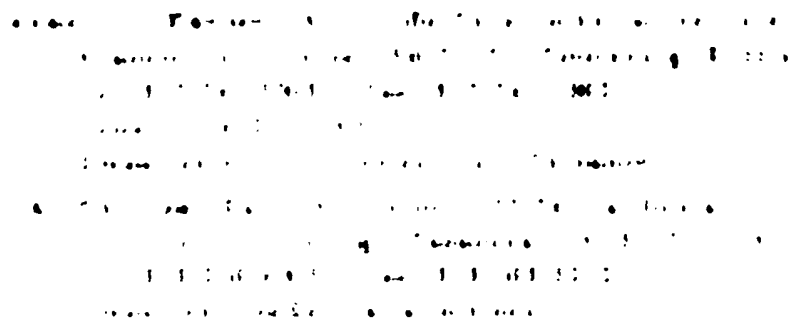

